\title{
Pionniers de la photographie en Suisse romande - Collection Auer Ory
}

27 septembre 2019-29 mars 2020, Maison Tavel

\section{Alexandre Fiette}

\section{(2) OpenEdition}

\section{Journals}

Édition électronique

URL : http://journals.openedition.org/artefact/5957

DOI : $10.4000 /$ artefact. 5957

ISSN : 2606-9245

Éditeur :

Association Artefact. Techniques histoire et sciences humaines, Presses universitaires du Midi

Édition imprimée

Date de publication : 15 juillet 2020

Pagination : 285-291

ISBN : 978-2-8107-0691-4

ISSN : 2273-0753

Référence électronique

Alexandre Fiette, "Pionniers de la photographie en Suisse romande - Collection Auer Ory », Artefact [En ligne], 12 | 2020, mis en ligne le 21 décembre 2020, consulté le 23 décembre 2020. URL : http:// journals.openedition.org/artefact/5957 ; DOI : https://doi.org/10.4000/artefact.5957

\section{(c) (i) () $९$}

Artefact, Techniques, histoire et sciences humaines est mise à disposition selon les termes de la Licence Creative Commons Attribution - Pas d'Utilisation Commerciale - Pas de Modification 4.0 International. 


\section{Pionniers de la photographie en Suisse romande - Collection Auer Ory}

27 septembre 2019-29 mars 2020, Maison Tavel

\section{Un lieu d'histoire légitimé par la photographie}

Devenue Musée d'histoire urbaine et quotidienne de Genève en 1986, après une longue gestation de son projet de conversion dont l'idée a germé dès 1920, la Maison Tavel entretient des liens intimes avec la photographie. En effet, cette demeure urbaine médiévale aujourd'hui institution publique municipale est le fruit de la volonté de mettre en valeur les collections historiques locales dites du Vieux-Genève. Celles-ci comprennent à l'origine un fonds important de photographies documentaires auquel se joint celui initialement constitué par le Musée suisse de photographies documentaires ${ }^{1}$, pour former ce qui sera l'actuel Centre d'iconographie genevoise ${ }^{2}$.

Si les expositions temporaires ne sont pas exclusivement consacrées à ce médium ni aux questions qui s'y rapportent, elles ont comporté de nombreux rendez-vous appréciés des visiteurs avec celui-ci ${ }^{3}$. On y a plus souvent

1. Sohier, Baum-Cousam, 2015.

2. Ce dernier a aujourd'hui quitté le groupement des musées municipaux genevois pour rejoindre celui des sites de la bibliothèque de Genève, sans que cela ne change leur statut commun d'institution culturelle de la Ville de Genève.

3. Parmi les diverses expositions, les volets successifs des Quartiers de mémoire, réalisés par Livio Fornara, ancien conservateur du Centre d'iconographie genevoise et de la Maison Tavel, ont laissé un vif souvenir à la population genevoise.

99 Alexandre Fiette, « Pionniers de la photographie en Suisse romande - Collection Auer Ory. 27 septembre 2019-29 mars 2020, Maison Tavel », Artefact, 12, 2020, p. 285-291. 
fait appel à l'indéniable charge sensible ou esthétique de l'image photographiée qu'à la technique ou l'exercice du procédé, difficile à imaginer dans le cadre d'un lieu dont la définition première et large de musée d'histoire locale ne draine pas un public dont l'intérêt pour la photographie et la connaissance des modalités de l'argentique seraient obligatoirement avérés. Pourtant, et c'est là toute l'ambiguïté, la photographie est considérée déjà depuis longtemps comme une pratique démocratique. Sa dématérialisation, dans les débuts du Xxi ${ }^{\text {e }}$ siècle, n'a fait que rapprocher chacun de l'acte de photographier en surmontant la technique jusqu'à désormais l'oublier et devenir de compulsifs collectionneurs d'images qui étonneraient les premiers photographes. C'est à partir de cette observation que l'exposition Les pionniers de la photographie en Suisse romande a trouvé son développement muséographique (Fig. 1).

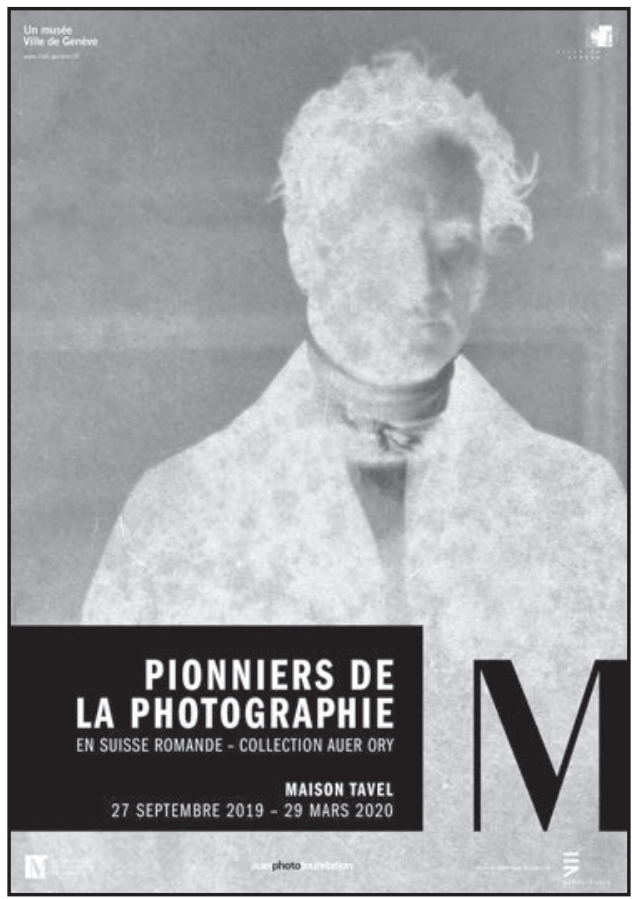

Fig. 1. - Affiche de l'exposition Pionniers de la photographie en Suisse romande - Collection Auer Ory

Juan Cris Pérez, Création d'Images, Genève 


\section{Une collection mise à disposition}

Michèle et Michel Auer, couple de collectionneurs bien connus du monde de la photographie, sont à l'origine du projet. En parallèle aux expositions généralement monographiques qu'ils réalisent au sein de leur fondation située à Hermance dans le canton de Genève, ils s'impliquent dans de nombreuses actions hors les murs de valorisation de leurs fonds illustrant tous les aspects de la photographie depuis ses débuts ${ }^{4}$. Cette fois, c'est autour de la période de développement de la pratique photographique, dans le cadre de la Suisse romande, qu'ils ont choisi de formuler leur proposition pour la Maison Tavel, réunissant pour la première fois le corpus d'œuvres liées à la Romandie qu’ils ont constitué depuis le début des années 1960.

Concevoir une exposition à partir d'une collection dont la seule raison d'être est la volonté d'une ou plusieurs personnes de grouper des pièces selon un schéma de pensée qui leur est propre, pose un cadre très différent de celui que donne l'exploitation d'un ou de multiples fonds, souvent institutionnels, dont la cohérence sacrifie rarement à l'émotion, au ressenti, à la recherche de pertinence qui motivent le collectionneur. Ce dernier est l'âme de sa collection et cela est doublement vrai avec Michèle et Michel Auer qui ont chacun façonné par leurs aspirations, choix et goûts, les contours de l'important ensemble qu'ils ont dédié à la photographie. Impossible donc d'aller chercher selon une thématique des ouvres qui l'illustrent sans casser ce fil qui les relie ; il faut plutôt considérer ce qui les lie pour en dégager des propos. Ce cheminement implique alors la compréhension du tout, donnant ainsi le moyen de respecter l'esprit de la collection, son entité, son identité (Fig. XIV, cahier couleur). De plus, la richesse du fonds mis à disposition rendait inutiles les spéculations sur la nécessité d'emprunts complémentaires d'une autre provenance qui auraient pu faire perdre la vision des deux collectionneurs.

\section{De l'unique au multiple}

S’il faut résumer les problématiques de la photographie naissante et synthétiser les axes de compréhension technique de cette dernière, c'est sans

4. Appareils, tirages, ouvrages d'artistes, publications et documentation relative à la photographie constituent leur très vaste collection. 
conteste par le rapprochement du daguerréotype au calotype que l'exercice prend tout son sens. Le premier est ce " plus parfait des dessins » vanté par Louis Daguerre (1787-1851) qui brevette l'invention portant son nom, développée à partir des recherches de Nicéphore Niépce (1765-1833) et de leurs travaux communs. Associés dès 1829 , ils mettent au point en 1932 le physautotype, image positive sur plaque d'argent enduite d'un vernis photosensible. Le daguerréotype sera lui aussi une image positive mais se formant après exposition sur un dépôt d'argent recouvrant une plaque généralement de cuivre, rendue sensible par des vapeurs d'iode, et développée par des vapeurs de mercure. Plus rapide, pérenne, d'un réalisme jusque-là inconnu, le procédé permet à Daguerre de cueillir les fruits de recherches antérieures faisant ainsi oublier le nom de Niépce décédé en 1833. Le daguerréotype, toujours sous un verre de protection ${ }^{5}$ pour protéger la fine couche d'argent, et souvent dans un écrin, connaît un grand engouement et l'on ne se désintéresse que vers 1855 de ces images dont les exemplaires ne peuvent être qu'uniques. À ce dernier va donc se confronter le calotype, ou Talbotype si l'on se réfère à William Henry Fox Talbot qui met au point le procédé. Il ne concurrence pas, à son apparition, la netteté et la précision de celui de Daguerre, mais n'en est pas moins porteur d'un plus grand potentiel. Le calotype permet en effet une répétition de l'image et ouvre ainsi la voie à une plus grande démocratisation de la photographie qui en exploite le principe jusqu'au passage à sa dématérialisation par la codification numérique autour de 2005. Sur un papier sensibilisé à l'iode de potassium et une solution de nitrate d'argent, l'image en négatif est développée, après exposition, dans une solution d'acide gallique et de nitrate d'argent, puis fixée. Souvent ciré pour lui donner de la transparence, ce négatif permet de répéter l'opération par contact, sur un papier traité par imprégnation d'une solution de sel et de nitrate d'argent et d'obtenir ainsi un positif. Les fibres du papier interférant avec la lumière, l'image est diffuse, problème que vont résoudre les négatifs verre recevant sur une face le collodion qui contient les éléments sensibles, ainsi que les papiers dits albuminés mis au point par Blanquart-Evrard en 1850 pour lesquels l'albumine du blanc d'œuf devient le véhicule des réactifs. Des techniques produisant des images uniques, le daguerréotype représente le premier et le plus saisissant des exemples, dont l'aboutissement condamne

5. Scellé sur le pourtour, il protège la délicate couche d'argent des rayures et de l'action de l'air, oxydant. 
tout développement conduisant à des procédés nouveaux. Le calotype, lui, loin de sa netteté, s'imposera cependant comme une étape, perfectible, dans l'évolution de la photographie. Fox Talbot qui en dépose le brevet en 1841, envisage dès 1844 à travers la publication de son ouvrage The Pencil of Nature, tout ce qu'il peut apporter au domaine de l'image, considérant qu'il est instrument au service de la nature pour l'expression de la richesse de cette dernière et de sa dimension artistique. Nouvel art ou reproduction fidèle sont les deux vecteurs de pensée de la toute jeune photographie. En est-il autrement aujourd'hui ?

\section{Lecture sensible}

Notre relation à la photographie, aux images qu'elle nous offre et nous permet d'obtenir, est conditionnée par sa capacité d'évocation. Choc, attrait, mémoire, esthétique, elle s'adresse aux sentiments tout autant qu'elle les suscite. Qu'en est-il de notre regard sur la production de ces Romands parmi lesquels on trouve des pionniers amateurs comme professionnels qui s'attachent à représenter ce qui leur tient à cœur ? JeanGabriel Eynard nous donne par ses daguerréotypes l'image de la Genève patricienne, Auguste Garcin des portraits inspirés, Samuel Heer réalise portraits et vues de Lausanne, Paul Louis Vionnet documente le canton de Vaud comme Jean Walther qui a aussi photographié la Grèce. Gabriel de Rumine rapporte, lui, des images de cet Orient en vogue, tandis que le Fribourgeois Pierre Rossier voyage en Asie et les Geiser originaires de La Chaux-de-Fonds s'établissent en Algérie ${ }^{6}$.

Les propos de leurs daguerréotypes et tirages sur papiers salés ou albuminés sont divers. Souvenirs affectifs, leurs portraits photographiques fixent l'apparence de chacun à tous les âges de la vie et jusque dans la mort alors que seule l'image peinte ou dessinée le permettait. Ils sont également un moyen de valorisation par une mise en scène qui se codifie (Fig. XLVI, cahier couleur). La transformation des centres urbains retient les regards sur les témoignages de leur passé comme des chantiers de construction qui assurent leur futur. Tout comme l'architecture, les ouvres d'art trouvent en la photographie une possibilité de diffusion et rencontrent ainsi un public toujours plus large. Enfin, c'est un certain sentiment de l'identité

6. Auer, 2019. 
helvétique que porte dès ses débuts la photographie en Suisse romande. Elle se substitue en cela aux gravures que les touristes du Grand Tour, voyage initiatique à l'art et au monde, rapportaient de leur passage en Suisse, étape de leur périple. La haute montagne, les glaciers et cascades sont des sujets de choix. La nature se révèle dans ce qu' elle offre de surprenant, mais aussi d'artistique, voire romantique. On s'intéresse également à l'habitat typique et au monde rural, dans une vision proche de l'ethnographie. Les Suisses romands rapportent eux aussi des images des étapes lointaines de ces grands voyages initiateurs du tourisme. Ils font connaitre paysages, édifices et habitants de contrées distantes. Certains suivent des évènements et les documentent préfigurant ainsi le reportage photographique. La collecte de vues du monde entier, parfois en stéréoscopie, incontournable nouveauté dès $1852^{7}$, fournit le reflet et concrétise ce que l'on appréhendait jusque-là par les mots et les dessins, confrontant alors une réalité à l'éventuelle subjectivité des écrivains et des artistes.

Dès ses premiers pas, la photographie suisse romande, à l'instar de celle qui s'expérimente partout ailleurs dans cette période pionnière s'achevant vers 1865 , répond à des questionnements et des attentes qui sont restés d'actualité comme en témoignent les 270 pièces de la collection Auer Ory exposées à la Maison Tavel. Elle n’en comporte pas moins des spécificités, des talents, et se professionnalisera rapidement.

\section{Alexandre Fiette Conservateur responsable de la Maison Tavel, Musées d'art et d'histoire, Genève}

\footnotetext{
7. Deux vues d'un même sujet prises avec un décalage latéral et placées devant les lentilles de l'appareil visionneur se recomposent dans l'œil humain avec effet de relief. L'enthousiasme de la reine Victoria à l'Exposition universelle de Londres pour le stéréoscope de Duboscq ne sera pas étranger à son succès.
} 
Pionniers de la photographie en Suisse romande - Collection Auer Ory

\section{Bibliographie}

La photographie des origines au début du XXe siècle. Vente aux enchères, Genève, mardi 13 juin 1961, Nicolas Rausch S. A. Photographies anciennes, appareils de photographie 1839-1900.

Auer Michel et Auer Michèle, Pionniers romands de la photographie, édition Fondation Auer Ory, Hermance, 2019.

$D u$, numéro 6 (Frühe Schweizerische Photos), Conzett et Huber, Zürich, juin 1952.

Fox Тацвот William Henri, The Pencil of Nature, Longman, Brown, Green and Longmans, Londres, 1844.

Sohier Estelle, Baume-Cousam Ursula, "Musée, histoire et photographie, le cas de Genève : sur les traces du Musée suisse de photographies documentaires (1901-1909)", dans Lacoste Anne, Corsini Silvio, Lugon Olivier (dir.), La Mémoire des images. Autour de la Collection iconographique vaudoise, Infolio, [s.l.], 2015, p. 168-193, https://archive-ouverte.unige.ch/unige:78295. 